\title{
Relato do uso de Clozapina em 56 pacientes atendidos pelo Programa de Atenção à Esquizofrenia Refratária da Secretaria da Saúde e do Meio Ambiente do Estado do Rio Grande do Sul
}

\author{
Clarissa Severino Gama* \\ Camila Morelatto de Souza** \\ Maria Inês Lobato* \\ Paulo Silva Belmonte de Abreu***
}

\section{INTRODUÇÃO}

\section{Epidemiologia}

A Esquizofrenia constitui o mais comum dos transtornos psiquiátricos graves. Aproximadamente, 20 milhões de pessoas sofrem dessa doença no mundo. A incidência anual de esquizofrenia é de 2 a 4 por 10.000 indivíduos com idade entre 15 e 54 anos, não havendo diferenças significativas entre os países ${ }^{1}$. Homens e mulheres são igualmente afetados ${ }^{2}$.

Estudo realizado no Serviço de Psiquiatria e Medicina Legal do HCPA (Rua: Ramiro Barcelos, 2350 - Porto Alegre).

* Médica psiquiatra do Programa de Esquizofrenia e Demências do Hospital de Clínicas de Porto Alegre (HCPA), Mestre em Medicina: Clínica Médica Universidade Federal do Rio Grande do Sul (UFRGS), membro do Comitê de Peritos em Medicamentos Psicoativos da Coordenação da Política de Atencão Integral à Saúde Mental da Secretaria Estadual da Saúde do Estado do Rio Grande do Sul.

** Doutoranda da Faculdade de Medicina da UFRGS.

*** Médico psiquiatra, Professor Adjunto do Departamento de Psiquiatria e Medicina Legal da UFRGS, Coordenador do Programa de Esquizofrenia e Demências do HCPA - Serviço de Psiquiatria, membro do Comitê de Peritos em Medicamentos Psicoativos da Coordenacão da Política de Atenção Integral à Saúde Mental da Secretaria Estadual da Saúde do Estado do Rio Grande do Sul.

\section{Definição e Manifestações:}

A esquizofrenia é difícil de ser definida ou descrita. Não existem traços patognomônicos, mas sintomas característicos que indicam uma desorganização da personalidade do paciente. A doença interfere, de uma forma geral, nos pensamentos, emoções, motivações e no comportamento motor da pessoa ${ }^{3,4}$.

Os sintomas psicóticos comprometem a capacidade de trabalho e de relações interpessoais por toda a vida ${ }^{5}$. Os sintomas podem ser divididos em dois grandes grupos: positivos ou floridos e negativos ou defeituosos. Os primeiros são exacerbações de funções normais ou anormalidades e distorções e incluem delírios e alucinações, hiperatividade e hostilidade. Os sintomas negativos incluem discurso pobre, isolamento social, avolia, desmotivação e embotamento emocional ${ }^{3,4}$. Alucinações, delírios, processos de pensamentos ilógicos ou incomuns e déficit na expressividade emocional e no funcionamento psicossocial são os sintomas mais freqüentes ${ }^{5}$

O quadro clínico é variável, podendo começar gradual ou repentinamente. Todavia, seu

Recebido em 04/11/2003. Revisado em 18/11/2003. Aprovado em 16/12/2003. 
curso é freqüentemente crônico e reincidente. Ele oscila entre remissão completa e deterioração mental grave, com a maioria dos pacientes sofrendo de incapacidade em níveis distintos. A doença acarreta importantes déficits psicológicos, sociais e vocacionais ${ }^{2}$.

\section{Tratamento e Prevenção}

A farmacoterapia tem provado ser o ponto chave na terapêutica da esquizofrenia. Embora não curativas, as drogas antipsicóticas (i.e. neurolépticas) estabeleceram-se como o tratamento primário para todos os estágios da doença. Reduzem o tempo de hospitalização e possibilitam o manejo continuado eficaz em seus lares.

As drogas antipsicóticas clássicas podem ser classificadas de alta, média e baixa potência. Quando usadas em doses eqüipotentes, todas as classes antipsicóticas parecem ter a mesma eficácia. A escolha da droga é feita, então, levando em consideração quais efeitos adversos serão menos prejudiciais a um determinado paciente 6 .

Durante um ataque agudo de esquizofrenia, o objetivo do tratamento é controlar os sintomas psicóticos positivos. Para a maioria dos pacientes que respondem rapidamente aos antipsicóticos, a melhora é observada em duas semanas, com o incremento ocorrendo em três ou quatro semanas, dependendo da dose. MeIhora adicional é vista dentro de alguns meses. É do conhecimento geral que os efeitos terapêuticos dos antipsicóticos são mais pronunciados nos sintomas agudos, positivos, do que nos sintomas crônicos, negativos, da esquizofrenia.

O tratamento de manutenção visa a manter os ganhos obtidos durante o tratamento agudo e a prevenir possíveis exacerbações da psicose e/ou readmissão hospitalar. O tratamento sintomático tem melhorado com a introdução da terapia antipsicótica, mas o prognóstico varia muito, tornando difícil alguma previsão².

Embora a eficácia dos antipsicóticos em amenizar os episódios psicóticos agudos e retardar a reincidência esteja bem estabelecida, essas drogas não são terapias absolutas. Da mesma forma, seu uso não é desprovido de problemas. Problemas comuns com a medicação incluem: resposta antipsicótica incompleta em um número significativo de pacientes, baixa eficácia em reverter o mau funcionamento e o prejuízo no comportamento social e interpessoal associado aos sintomas negativos da esquizofrenia ${ }^{7}$ e o surgimento de sintomas extrapiramidais $^{8,9}$ e de toxicidade. Mais de $20 \%$ de todos os pacientes mantêm-se refratários ao tratamento com antipsicóticos clássicos ${ }^{1,10,11}$. Dos pacientes que estão no primeiro episódio de esquizofrenia, 14\% não responderão adequadamente $^{12}$. Aproximadamente, 20 a $30 \%$ dos pacientes que inicialmente respondem são passíveis de recaídas, mesmo com a manutenção da terapia antipsicótica ${ }^{13}$. Dessa forma, a despeito da grande eficácia das medicações antipsicóticas no tratamento da esquizofrenia, uma proporção substancial de pacientes continua a experimentar uma significativa psicopatologia clínica e a ter pobre prognóstico em longo prazo.

As estimativas da proporção de pacientes refratários variam consideravelmente, sendo influenciadas por fatores como fase da doença, definição de refratariedade e duração das experiências de tratamento. O reconhecimento desses problemas leva à definição de uma categoria denominada esquizofrenia resistente à terapia. Seis níveis de resposta ao tratamento foram definidos:

- Níveis 1, 2 e 3: respostas excelente, muito boa e boa, respectivamente.

- Nível 4: resposta satisfatória, que compreende a solução lenta e incompleta, mas com remissão clínica e social.

- Nível 5: resposta baixa, que compreende a não remissão a despeito do uso de medicação antipsicótica standard, impossibilitando a reabilitação. Esta categoria inclui os pacientes que abandonaram o tratamento por toxicidade ou efeitos indesejáveis.

- Nível 6: a resistência grave compreende a não remissão a despeito de tratamento medicamentoso em doses plenas, por tempo suficiente (seis meses), com cuidados intensivos no âmbito de internação hospitalar, como medidas seriadas de níveis plasmáticos e intervenção psicossocial intensiva. Exige a permanência no hospital ou cuidados semelhantes.

\section{Clozapina}

Apesar da freqüência elevada de sintomas persistentes em pacientes esquizofrênicos, poucos tratamentos alternativos têm surgido como auxílio efetivo nesses casos. O maior impulso no desenvolvimento de novos componentes tem sido separar os efeitos antipsicóticos das drogas antipsicóticas. A clozapina é a mais importante aquisição em terapia antipsicótica desde o advento da clorpromazina ${ }^{14}$.

Do ponto de vista farmacodinâmico, a clozapina tem atividade antagonista em receptores $5-\mathrm{HT}_{2 \mathrm{~A}}, \mathrm{D}_{1}, \mathrm{D}_{4}$, alfa adrenérgico, colinérgico 
e histamínicos ${ }^{15}$. O antagonismo em receptores $\mathrm{D}_{2}$, característico de antipsicóticos típicos, é menos proeminente com a clozapina ${ }^{16}$. Por ter características distintas das medicações até então disponíveis ${ }^{17}$, a clozapina é considerada um antipsicótico atípico ${ }^{18}$. Entre essas características, podemos citar a eficácia legítima em $30-61 \%$ das psicoses refratárias às abordagens com antipsicóticos típicos ${ }^{14,19-21}$ e a baixa incidência ou o adiamento das manifestações extrapiramidais e da discinesia tardia ${ }^{22,23}$. Também se observou melhora dos sintomas negativos (anergia, anedonia, avolição, embotamento de afeto) e das funções cognitivas e sociais e redução da agressividade ${ }^{14,19,24-26}$.

\section{MATERIAIS E MÉTODOS}

Em Janeiro de 1991, foi estabelecido convênio entre a Secretaria da Saúde e Meio Ambiente do Estado do Rio Grande do Sul e o Hospital de Clínicas de Porto Alegre com a finalidade de iniciar o fornecimento gratuito de clozapina aos pacientes com diagnóstico de Esquizofrenia ${ }^{27}$ Refratária. A Secretaria da Saúde e do Meio Ambiente do Estado do Rio Grande do Sul comprometeu-se a:

1. Realizar a pré-triagem dos casos e seu encaminhamento aos locais de atendimento;

2. Analisar laudos trimestrais sobre a demanda dos locais de atendimento, visando à previsão de gastos;

3. Distribuir a medicação, conforme sua possibilidade.

Aos locais de atendimento, entre eles o Hospital de Clínicas de Porto Alegre, coube:

1. Avaliar o paciente a partir dos critérios de inclusão previamente definidos;

2. Encaminhar o laudo inicial informando sobre o paciente, firmando ou excluindo o diagnóstico de Esquizofrenia Refratária e, conseqüentemente, auxiliando na decisão sobre a participação do paciente no programa;

3. Garantir a continuação do atendimento ambulatorial destes pacientes;

4. Elaborar laudos trimestrais com a evolução de cada paciente beneficiado.

Este artigo é, portanto, o relato da experiência clínica obtida através do Programa de Atenção à Esquizofrenia Refratária com prescrição de Clozapina, no período de abril de 1991 a julho de 1999. Tem por objetivo avaliar a resposta dos sintomas psicóticos ao uso da clozapina, usando a escala Brief Psychiatric Rating Scale (BPRS) ${ }^{28}$ como método de aferição. Envolveu, de maneira consecutiva, os primeiros cinqüenta e seis pacientes que rece- biam atendimento no Ambulatório do Programa de Esquizofrenia e Demências do Serviço de Psiquiatria do Hospital de Clínicas de Porto Alegre, que preencheram os seguintes critérios de inclusão do programa:

1. Falhar em mostrar resposta ao tratamento com haloperidol em doses orais de $20-40 \mathrm{mg} /$ dia durante um período de 12 semanas;

2. Falhar em responder ao tratamento com clorpromazina oral 1 g/dia por 12 semanas;

3. Não mostrar resposta ao tratamento com Tioridazina 400-800 mg/dia por 24 semanas;

4. Não apresentar nenhum período de funcionamento pessoal adequado nos últimos 2 anos;

5. Apresentar presença continuada de sintomatologia grave nos últimos dois anos.

Os pacientes foram acompanhados em três momentos distintos:

1. Nas primeiras dezoito semanas, foram vistos semanalmente para ajuste medicamentoso, controle hematológico e verificação de sintomas;

2. A partir da décima nona semana, os pacientes foram acompanhados mensalmente para reajuste de dose e verificação de sintomas e controle hematológico;

3. A BPRS foi realizada trimestralmente.

Foram excluídos os seguintes pacientes:

1. Ausência de família organizada e participante;

2. História de epilepsia precedendo o diagnóstico de esquizofrenia;

3. História de tumor cerebral;

4. Presença de doença grave hepática, renal ou cardíaca;

5. Psicose alcoólica ou tóxica;

6. História de dependência/abuso de drogas psicoativas; tação.

7. Situação potencial de gravidez e/ou lac-

O tratamento foi interrompido nos pacientes em que houve falha em mostrar resposta adequada ao tratamento após o uso ininterrupto por 12 semanas em doses de $300-800 \mathrm{mg}$ ao dia, via oral. Não resposta ao tratamento é definida como uma diminuição inferior a $20 \%$ na escala BPRS ${ }^{29}$

\section{Análise Estatística}

A análise estatística foi executada pelo programa estatístico Statistical Product and Service Solutions (SPSS) versão 8.0. Foi usado o teste de Mann-Whitney U para comparações não paramétricas. O coeficiente de Regressão Linear Simples foi utilizado para estudo de rela- 
ção da idade, escolaridade, número de internações, idade na primeira internação e tempo de doença prévio ao uso de clozapina com a variação entre o BPRS antes do início do tratamento e o BPRS medido aos 93 meses de tratamento.

\section{RESULTADOS}

Quarenta pacientes eram do sexo masculino e 16 do sexo feminino. Estudaram, em média, nove anos com desvio padrão de 3 anos. A idade de início do tratamento com clozapina, o número de internações prévias, a idade na primeira internação, o tempo de doença e a dose de clozapina usada são apresentados na Tabela 1. A média dos escores da BPRS antes do tratamento com clozapina foi de 77,9 $(\mathrm{DP}=16,1)$; após 6 meses do início do tratamento, foi de 44,4 $(\mathrm{DP}=16,4)$ e após 93 meses de início do tratamento, foi de 41,1 ( $D P=16,2)$. As diferenças entre a média do escore na BPRS antes do tratamento e após 6 meses de início do tratamento e antes do tratamento, comparadas com a média na BPRS após 93 meses de início do tratamento, foram estatisticamente significativas ( $p<0,01$, para ambas comparações). A média da variação dos escores na escala BPRS antes do uso da clozapina e nos primeiros 93 meses de tratamento pode ser vista na Figura 1. Dois pacientes não tiveram seus escores clínicos avaliados trimestralmente por serem institucionalizados, sendo os dados dos 54 pacientes restantes avaliados nesses itens.

Tabela 1. Características da amostra (média \pm DP)

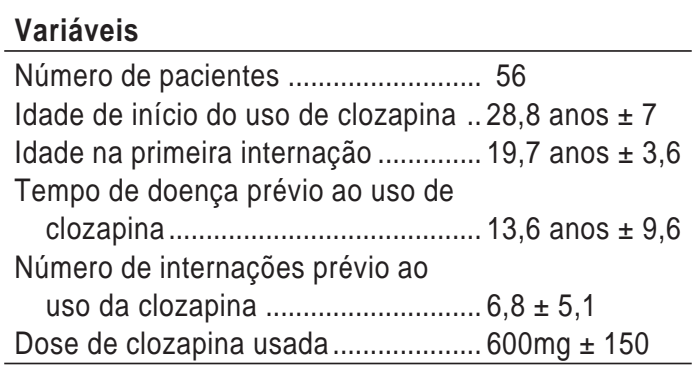

Sete pacientes tiveram convulsões após o início do uso da clozapina (12\%); nenhum deles teve o tratamento interrompido por esta razão. As convusões foram tratadas com anticonvulsivantes. A dose média de clozapina usada foi de $600 \mathrm{mg}(\mathrm{DP}=150)$, sendo que esta diminui para $550 \mathrm{mg}(\mathrm{DP}=150)$ no grupo de pacientes que não apresentou convulsões e aumenta para $700 \mathrm{mg}(\mathrm{DP}=100)$ naqueles que apresentaram

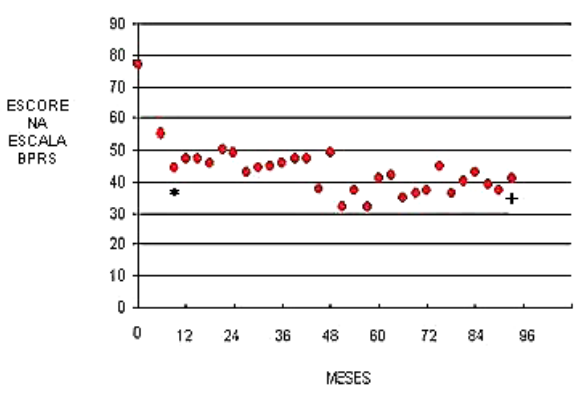

Figura 1. Média da variação dos escores trimestrais na escala BPRS * $p \leqslant 0,01$ antes do tratamento vs. 6 meses de tratamento (Mann-Mhitney U test) $+p \leqslant 0,01$ antes do tratamento vs. 93 meses de tratamento (Mann-Whitney U test)

convulsões $(p=0,04)$. Dois pacientes abandonaram o programa e um foi excluído por agranulocitose (2\%). Durante o período de abril de 1991 e julho de 1999, houve quatro internações hospitalares neste grupo de pacientes $(0,07$ por paciente), sendo um paciente internado duas vezes e dois pacientes internados uma vez.

A variável tempo de doença prévio ao uso de clozapina mostrou relação inversa com a diferença entre o BPRS antes do início do tratamento e o BPRS medido aos 93 meses de tratamento. Quanto maior o tempo de doença prévio ao uso de clozapina, menor a diferença entre as medidas de BPRS. As variáveis escolaridade, número de internações e idade na primeira internação não tiveram relação com a diferença entre o BPRS antes do início do tratamento e o BPRS medido aos 93 meses de tratamento (tabela 2).

Tabela 2. Relação preditiva entre as variáveis individuais e a diferença entre o BPRS antes do início do tratamento e o BPRS medido aos 93 meses de tratamento (regressão linear simples): R (IC. 95\%)

\begin{tabular}{l} 
Variáveis $\quad$ R (IC 95\%) \\
\hline Idade ................................ $-0,51(\mathrm{IC}-0,07 ; 0,89)$ \\
Escolaridade .................. $-0,45(\mathrm{IC}-0,73 ; 0,87)$ \\
Número de internações ...... $-0,54(\mathrm{IC}-0,68 ; 0,89)$ \\
Idade na primeira \\
$\quad$ internação ..................... - $0,36(\mathrm{IC}-0,76 ; 0,85)$ \\
Tempo de doença prévio ao uso de \\
clozapina ......................... $-0,75(\mathrm{IC}-0,95 ;-0,39)^{*}$ \\
${ }^{*} \mathrm{p}<0,05$
\end{tabular}

\section{DISCUSSÃO}

Estudos clínicos têm demonstrado que a clozapina é eficaz na supressão tanto de sintomas positivos quanto negativos da esquizofrenia e está associada com uma incidência muito baixa de efeitos adversos extrapiramidais ${ }^{30-32}$. Além disso, é eficaz em uma proporção subs- 
tancial (30 a 60\%) de pacientes esquizofrênicos que não respondem ou não toleram a terapia com drogas antipsicóticas clássicas. Outros estudos mostram que pacientes com esquizofrenia refratária tratados com clozapina por períodos prolongados (seis meses a dois anos) ${ }^{33-}$ 35 obtiveram melhora na adaptação social, integração e funções cognitivas ${ }^{36-39}$, remissão substancial da doença e conseqüentemente maior afastamento das internações hospitalares, melhor qualidade de vida e potencial de trabalho $37,40-45$.

Tais resultados confirmam a eficácia desse fármaco em suprimir ou atenuar tanto os sintomas negativos quanto as alterações cognitivas, fazendo com que haja, de fato, uma alternativa farmacológica que possibilite ao paciente retomar a sua vida com melhor qualidade. Foi encontrada uma diminuição estatisticamente significativa dos escores no BPRS nos pacientes deste estudo após 6 meses do início do uso de clozapina, fato que confirma os achados da literatura quanto à eficácia deste fármaco na diminuição dos sintomas positivos e negativos da esquizofrenia em pacientes refratários. Segundo recente estudo clínico ${ }^{29}$, é considerada resposta à clozapina uma diminuição maior que $20 \%$ do escore inicial na BPRS, fato constatado nos pacientes deste estudo.

Quanto à reabilitação do paciente esquizofrênico, é importante salientar que os sintomas negativos (alogia, embotamento afetivo, anedonia e avolição) tendem a prejudicar a capacidade da pessoa de levar uma vida cotidiana normal. Estes impedem que os pacientes mantenham relacionamentos familiares normais, freqüentem escolas, tenham um emprego ou formem amizades e relacionamentos íntimos. Dessa forma, impõem um prejuízo substancial à qualidade de vida desses indivíduos, sobretudo porque se tornam mais graves com o tempo. Além dos sintomas negativos, o déficit cognitivo sofrido por esses pacientes é também responsável por dificuldades de relacionamento social e íntimo, bem como diminuição da capacidade para o trabalho. Em decorrência disso, a remissão ou melhora dos sintomas negativos e cognitivos passou a ser uma das preocupações na terapêutica dos pacientes esquizofrênicos, na tentativa de lhes proporcionar convívio familiar e social mais adequados, assim como possibilitar o exercício de atividade profissional rentável.

Apesar das baixas taxas de manifestações extrapiramidais (4-7\%), o tratamento com clozapina está associado a uma vasta gama de efeitos adversos ${ }^{16,44}$. A agranulocitose, a neu- tropenia e a leucopenia têm incidência variando entre $0,05 \%$ a $2,8 \%$; na amostra estudada, um paciente teve agranulocitose, correspondendo a $2 \%$ do total, não diferindo do apresentado na literatura. Esta incidência varia conforme características individuais de cada paciente, dosagem e duração de tratamento. Na grande maioria, ocorrem nos seis meses iniciais de tratamento, com $75-80 \%$ ocorrendo nas $4-18$ semanas iniciais ${ }^{19}$. Os pacientes também apresentam comumente hipotensão ortostática (por bloqueio alfa adrenérgico), taquicardia, visão turva, xerostomia, hipersalivação, constipação e sedação devido à ação anticolinérgica, porém há marcada tolerância a estas manifestações ${ }^{16,19}$. Casos de convulsões variam de 1 $10 \%$ e são descritas como dose-dependentes ${ }^{16}$. Os achados desta amostra (12,5\%) são concordantes com o da literatura quanto à prevalência dos casos de convulsão e do seu aparecimento estar relacionado ao aumento da dose de clozapina.

Alguns indicadores de prognóstico para resposta à clozapina foram idade jovem, paciente refratário, início precoce da doença e longa história de doença. Os pacientes paranóides e com maior nível inicial de sintomas extrapiramidais têm melhor resposta ao tratamento $^{19}$. Encontramos nesta amostra que o tempo de doença prévio ao uso de clozapina pode ser um indicador de prognóstico para a resposta a este fármaco, ou seja, podem vir a apresentar pior resposta à clozapina aqueles com mais tempo de doença antes do seu uso.

O tratamento do paciente esquizofrênico tem-se modificado nos últimos tempos, podendo-se citar: (a) desinstitucionalização; (b) meIhora de tratamento na comunidade; (c) busca de otimização e racionalização de custos e recursos em saúde; (d) desenvolvimento de rotinas e padronização de recursos diagnósticos multiaxiais e de procedimentos; (e) montagem de programas de reabilitação, educação da família e do paciente sobre a doença e as formas de manejá-la ${ }^{45}$. Considerando o número de internações por paciente antes do uso da clozapina $(6,8)$ e depois $(0,07)$, nesta amostra, vê-se claramente o benefício deste fármaco em ajudar a manter o paciente na comunidade. Dessa forma, a clozapina ${ }^{46}$ tornou-se o tratamento alternativo melhor estabelecido para pacientes refratários.

\section{CONCLUSÃO}

Observando os resultados apresentados, podemos concluir que os pacientes apresenta- 
ram uma melhora significativa da sintomatologia avaliada pelo BPRS após seis meses de início do tratamento e que esta melhora se mantém após noventa e três meses de início do tratamento com clozapina. Além disso, um maior tempo de evolução da doença antes do uso da clozapina, em pacientes refratários, pode ser preditor de menor resposta ao tratamento, corroborando a hipótese de que um maior tempo de exposição à psicose está correlacionado com modificações neurofisiológicas e neuroquímicas desfavoráveis, envolvendo eventualmente processos cerebrais degenerativos $^{47}$. Vale ressaltar a importância do início precoce do tratamento da psicose e da necessidade da participação do Estado, no sentido de oferecer suporte psicossocial e financeiro para a otimização do tratamento de pacientes psiquiátricos.

Em um primeiro momento, os pacientes beneficiados eram atendidos, exclusivamente, no Hospital de Clínicas de Porto Alegre, Hospital Psiquiátrico São Pedro, Hospital MaternoInfantil Presidente Vargas e no Hospital da Faculdade de Medicina de Santa Maria. A partir de julho de 1999, o fornecimento de clozapina pela Secretaria da Saúde do Estado do Rio Grande do Sul expandiu-se para todo paciente que preenchesse critérios para inclusão no programa e que realizasse seu tratamento no Rio Grande do Sul, em âmbito público ou privado. Atualmente, cerca de 500 pacientes estão se beneficiando com esse programa.

\section{REFERÊNCIAS BIBLIOGRÁFICAS}

1. Sartorius N, Jablensky A, Korten A, Ernberg G, Anker M, Cooper JE, et al. Early manifestations and first-contact incidence of schizophrenia in different cultures. A preliminary report on the initial evaluation phase of the WHO Collaborative Study on determinants of outcome of severe mental disorders. Psychol Med 1986;16:909-28.

2. Kaplan HI, Sadock BJ, Grebb JA. Esquizofrenia. In: Kaplan HI, Sadock BJ, Grebb JA, editors. Compêndio de Psiquiatria - Ciências do Comportamento e Psiquiatria Clínica. $7^{\mathrm{a}} \mathrm{ed}$. Porto Alegre: Artmed; 1985. p. 439-66.

3. Andreasen NC. Negative symptoms in schizophrenia. Definition and reliability. Arch Gen Psychiatry 1982;39:784 8.

4. Andreasen NC, Olsen S. Negative v. positive schizophrenia. Definition and validation. Arch Gen Psychiatry 1982;39:789-94.

5. Levinson DF. Pharmacologic treatment of schizophrenia. Clin Ther 1991;13:326-52.

6. Marder SR, Mintz J, Van Putten T, Lebell M, Wirshing WC, Johnston-Cronk K. Early prediction of relapse in schizophrenia: an application of receiver operating characteristic (ROC) methods. Psychopharmacol Bull 1991;27:79-82.

7. Meltzer HY. Biological studies in schizophrenia. Schizophr Bull 1987;13:77-111.
8. Jeste DV, Wyatt RJ. Dogma disputed: is tardive dyskinesia due to postsynaptic dopamine receptor supersensitivity? J Clin Psychiatry 1981;42:455-7.

9. Klawans HL, Goetz CG, Perlik S. Tardive dyskinesia: review and update. Am J Psychiatry 1980;137:900-8.

10. Davis BD. Frontiers of the biological sciences. Science 1980;209:78-89

11. Haring $C$, Fleischhacker WW, Schett $P$, Humpel C, Barnas C, Saria A. Influence of patient-related variables on clozapine plasma levels. Am J Psychiatry 1990;147:1471-

12. Lieberman JA, Saltz BL, Johns CA, Pollack S, Borenstein $\mathrm{M}$, Kane J. The effects of clozapine on tardive dyskinesia. Br J Psychiatry 1991;158:503-10.

13. Lieberman JA, Kane JM, Alvir J. Provocative tests with psychostimulant drugs in schizophrenia. Psychopharmacology (Berl) 1987;91:415-33.

14. Lieberman JA. Clinical effects of clozapine in chronic schizophrenia: response to treatment and predictors of outcome. Am J Psychiatry 1994;151:12.

15. Kahn RS, Davidson M, Siever L. Serotonin function and treatment response to clozapine in schizophrenic patients. Am J Psychiatry 1993;150:1337-42.

16. Meltzer HY. Measuring outcome in schizophrenia: differences among atypical antipsychotics. J Clin Psychiatry 1998;59:3-9.

17. Jann M. Clozapine. Pharmacotherapy 1991;11:179-195.

18. Lieberman JA, Kane JM, Johns CA. Clozapine: guidelines for clinical management. J Clin Psychiatry 1989;50:329-38

19. Buchanan RW. Clozapine: Efficacy and Safety. Schizophr Bull 1995;21:4.

20. Hofer A, Hummer M, Kemmler G, Kurz M, Kurzthaler I, Fleischhacker WW. The safety of clozapine in the treatment of first and multiple episode patients with treatmentresistant schizophrenia. Int J Neurosychopharmacol 2003;6:201-206.

21. Freedman R. Schizophrenia - Drug Therapy. N Engl J Med 2003;349:1738-49.

22. Kane JM, Margaret GW, Pollack, S. Does clozapine cause tardive dyskinesia? J Clin Psychiatry 1993;54:327-30.

23. Sacgdev P, Kruk J, Kneebone M. Clozapine-induced neuroleptic malignant syndrome: review and report of new cases. J Clin Psychopharmacol 1995;15:365-71.

24. Kane JM, Mayerhoff D. Do negative symptoms respond to pharmacological treatment? $\mathrm{Br} \mathrm{J}$ Psychiatry Suppl 1989;7:115-8.

25. Meltzer H. Novel Antipsychotic Drugs. Raven Press 1992;1:1-13.

26. Abreu PSB, Schestatsky S, Lobato MI. O uso da Clozapina em pacientes em pacientes esquizofrênicos. J Brás Psiquiatr 1995;44(2):59-62.

27. American Psychiatric Association. Diagnostic and Statistical Manual of Mental Disorders, fourth Edition. Washington (DC); 1994.

28. Thompson PA, Buckley PF, Meltzer HY. The brief psychiatric rating scale: effect of scaling system on clinical response assessment. J Clin Psychopharmacol 1994:14:344-6.

29. Mauri MC, Volonteri LS, Dell'Osso B, Regispani F, Papa $P$, Baldi M, Bareggi SR. Predictors of clinical outcome in schizophrenic patients responding to clozapine. J Clin Psychopharmacol 2003; 23(6):660-664.

30. Alphs L, Meltzer H. Side Effects of Clozapine and Their Management. Clin Neuropharmacol 1990;13:261-2.

31. Kane J, Honigfeld G, Singer J, Meltzer H. Clorazil collaborative study group - Clozapine for the treatment resistent schizophrenic: a double-blind comparison with clorpromazine. Arch Gen Psychiatry 1988;45:789-96. 
32. Owen RR, Beake BJ, Marby D. Response to Clozapine in Chronic Psychotic Patients. Psychopharmacol Bull 1989;25:253-6.

33. Pickar D, Owen RR, Litman RE. Clinical and biologic response to clozapine in patients with schizophrenia. Arch Gen Psychiatry 1992;49:345-53.

34. Lindenmayer JP, Grochowski S, Mabugat L. Clozapine effects on positive and negative symptoms: a six-month trial in treatment-refractory schizophrenics. J Clin Psychopharmacol 1994;14:201-4.

35. Remington GJ. The long-term treatment of schizophrenia. Can J Psychiatry 1995;40:1.

36. Meltzer HY, Burnett S, Bastani B, Ramirez LF. Effects of six month of clozapine treatment on the quality of life of chronic schizophrenic patients. Hosp Community Psychiatry 1990;41:892-97.

37. Meltzer HY, Cola P, Way L, Thompson PA, Bastani B, Davies MA, et al. Cost effectiveness of clozapine in neuroleptic-resistant schizophrenia. Am J Psychiatry 1993;150:1630-8.

38. Joffe G, Venalainen E, Tupala J, Hiltunen O, Wahlbeck K, Gadeke R, et al. The effect of clozapine on the course of illness in chronic schizophrenia: focus on treatment outcome in out-patients. Int Clin Psychopharmaco 1996;11:265-72.

39. Breier A, Buchanan RW, Irisch D, Carpenter WT. Clozapine treatment of outpatients with schizophrenia: outcome and long-term response patterns. Hosp Community Psychiatry 1993;44:1145-9.

40. Goldberg T, Greenberg R, Griffin S. The effect of clozapine on cognition and psychiatry symptoms in patients with schizophrenia. Br J Psychiatry 1993;162:43-8.

41. Honigfeld G, Patin J. A two-year clinical and economic follow-up of patients on clozapine. Hosp Community Psychiatry 1990;41:882-5

42. Meltzer HY, Cola PA. The pharmacoeconomics of clozapine: a review. J Clin Psychiatry 1994;55:161-5.

43. Meynard JA, Bernadet R, Sanchez MF, Morel P. Schizophrenia, affective symptoms and clozapine. Encephale 1995;21:41-7.

44. Llorca PM, Lancon C, Auquier P, Bougerol T. Clinical effects of clozapine: effect on negative symptoms. Encephale 1996;22:287-92.

45. Weiden PJ, Olfson M. Cost of relapse in schizophrenia. Schizophr Bull 1995;21:419-29.

46. Jalenques I, Coudert AJ. A new therapeutic approach tp drug-resistant schizophrenia: clozapine. Long-term pros pective study in 16 patients. Acta Psychiatr Belg 1992;92:323-38.

47. Lieberman JA. Pathophysiologic mechanisms in the pathogenesis and clinical course of schizophrenia. Journal of Clinical Psychiatry 1999; 60: S9-S12.

\section{RESUMO}

Introdução: A Esquizofrenia é transtorno psiquiátrico crônico grave que acarreta importantes déficits psicológicos, sociais e vocacionais. Os antipsicóticos clássicos são amplamente usados como tratamento, mas comumente produzem respostas incompletas, toxicidade e efeitos extrapiramidais. A clozapina foi importante aquisição na terapia antipsicótica. Mostrou-se com eficácia legítima em 30-61\% das psicoses e baixa incidência de efeitos adversos. Métodos: Relato da experiência clínica de 56 pacientes com Esquizofrenia Refratária incluídos no programa de fornecimento gratuito da clozapina pela Secre- taria da Saúde e do Meio Ambiente do Estado do Rio Grande do Sul em convênio com o Hospital de Clínicas de Porto Alegre. Resultados: A média dos escores da Brief Psychiatric Rating Scale (BPRS) foi inicialmente $77,9(D P=16,1)$ e, após noventa e três meses de tratamento, 41,1 (DP=16,2). Dois pacientes abandonaram o programa e um foi excluído por agranulocitose. Houve quatro internações. Discussão: Apesar de sua comprovada eficácia e aplicabilidade, a clozapina não está livre de efeitos adversos: hipotensão ortostática, taquicardia, visão turva, xerostomia, hipersalivação, constipação e sedação são comuns. As alterações hematológicas ocorrem em 0,05 a 2,8\% dos casos. Ainda, o alto custo restringe seu uso. Conclusões: Houve melhora significativa e mantida dos pacientes que participaram do programa. Doenças com maior tempo de evolução obtiveram menor resposta, provavelmente relacionada a alterações neurofisiológicas e neuroquímicas. Vale ressaltar a importância do início precoce do tratamento e a necessidade da participação do Estado, no sentido de oferecer suporte psicossocial e financeiro para a otimização do tratamento desses pacientes.

Descritores: Clozapina, esquizofrenia refratária, saúde pública.

\section{ABSTRACT}

Introduction: Schizophrenia is a chronic severe psychiatric disease causing major psychological, social and working deficiencies. Typical neuroleptics are widely used, but often produce incomplete responses, toxicity and adverse effects on motricity. Clozapine was a significant development. It is effective in treating $30-61 \%$ of the psychotic symptoms with minimal adverse effects. Methods: Clinical experience of 56 patients with refractory schizophrenia under treatment at the Psychiatry Service of the Hospital de Clínicas de Porto Alegre, included in the program to supply clozapine free of charge, promoted by the Department of Health and the Environment of the State of Rio Grande do Sul. Results: The mean score for the Brief Psychiatric Rating Scale (BPRS) was initially $77.9(S D=16.1)$ and, at the end, 41.1 $(S D=16.2)$. Two patients left the program and one was excluded because he developed agranulocytosis. There were 4 hospitalizations. Discussion: Despite its well-established efficacy and applicability, clozapine is not free from adverse effects: postural hypotension, tachycardia, cloudy vision, dry eyes, hypersalivation, constipation and sedation frequently occur. Hematological alterations occur in 0.05-2.8\%. Conclusions: There was a significant and lasting improvement of the symptoms in the patients enrolled. Diseases with a longer evolution had worse responses, probably related to neurochemical and neurophysiological damage. The importance of early treatment and the need for State intervention, offering psychosocial and financial support to optimize the treatment of this population should be highlighted. 
Keywords: Clozapine, refractory schizophrenia, public health.

Title: Clozapine use report in 56 Patients Seen by Clerkship of Health and Environment of the State of Rio Grande do Sul's Program of Attention to the Refractory Schizophrenia

\section{RESUMEN}

Introducción: La Esquizofrenia es un trastorno psiquiátrico crónico grave que ocasiona importante déficit psicológico, social y vocacional. Los antipsicóticos clásicos son ampliamente usados como tratamiento, pero comunmente producen respuestas incompletas, toxicidad y efectos extrapiramidales. La clozapina fue importante adquisición en la terapia antipsicótica. Mostró legítima eficacia en 30-61\% de las psicosis y baja incidencia de efectos adversos. Métodos: Relato de la experiencia clínica de $56 \mathrm{pa}$ cientes con Esquizofrenia Refractaria incluídos en el programa de suministro gratuito de clozapina por la Secretaría de Salud y Medio Ambiente del Estado de Rio Grande do Sul en convenio con el Hospital de Clínicas de Porto Alegre. Resultados: La media de los escores da Brief Psychiatric Rating Scale (BPRS) fue inicialmente $77,9(D P=16,1)$ y, después de noventa y tres meses de tratamiento, 41,1 $(D P=16,2)$. Dos pacientes abandonaron el programa y uno fue excluído por agranulocitosis. Hubo cuatro internaciones. Discusión: A pesar de su comprobada eficacia y aplicabilidad, la clozapina no está libre de efectos adversos: hipotensión ortostática, taquicardia, visión turbia, xerostomia, hipersalivación, constipación y sedación son comunes. Las alteraciones hematológicas ocurren en 0,05 a 2,8\% de los casos. Aún, el alto costo restringe su uso. Conclusiones: Hubo mejoría significativa y estable de los pacientes que participaron del programa. Enfermedades con mayor tiempo de evolución obtuvieron menor resultado, probablemente relacionada a alteraciones neurofisiológicas y neuroquímicas. Vale resaltar la importancia del inicio precoz del tratamiento y de la necesidad de participación del Estado, en el sentido de ofrecer soporte psicosocial y financiero para la optimización del tratamiento de estos pacientes.

Palabras-clave: Clozapina, esquizofrenia refractaria, salud pública.

Título: El relato del uso de Clozapina en 56 Pacientes Atendidos por el Programa de Atención a la Esquizofrenia Refractaria de la Secretaria de Salud y Medio Ambiente del Estado de Rio Grande do Sul

Endereço para correspondência:

Clarissa Severino Gama

Rua Passo da Pátria, 579/301

90460-060 - Porto Alegre - RS

E-mail:csgama@yahoo.com

Copyright (c) Revista de Psiquiatria do Rio Grande do Sul - SPRS 